ORIGINAL ARTICLE

\title{
Prospective noise induced changes to hearing among construction industry apprentices
}

\author{
N S Seixas, B Goldman, L Sheppard, R Neitzel, S Norton, S G Kujawa
}

Occup Environ Med 2005;62:309-317. doi: 10.1136/oem.2004.018143

See end of article for authors' affiliations .....................

Correspondence to: Dr N Seixas, University of Washington, Department of Environmental and Occupational Health Sciences, Seattle, WA, USA; nseixas@ u.washington.edu

Accepted 6 January 2005

\begin{abstract}
Aims: To characterise the development of noise induced damage to hearing.
Methods: Hearing and noise exposure were prospectively monitored among a cohort of newly enrolled construction industry apprentices and a comparison group of graduate students, using standard pure tone audiometry and distortion product otoacoustic emissions (DPOAEs). A total of 328 subjects (632 ears) were monitored annually an average of 3.4 times. In parallel to these measures, noise exposure and hearing protection device (HPD) use were extensively monitored during construction work tasks. Recreational/non-occupational exposures also were queried and monitored in subgroups of subjects. Trade specific mean exposure $L_{e q}$ levels, with and without accounting for the variable use of hearing protection in each trade, were calculated and used to group subjects by trade specific exposure level. Mixed effects models were used to estimate the change in hearing outcomes over time for each exposure group.

Results: Small but significant exposure related changes in DPOAEs over time were observed, especially at $4 \mathrm{kHz}$ with stimulus levels (L1) between 50 and $75 \mathrm{~dB}$, with less clear but similar patterns observed at $3 \mathrm{kHz}$. After controlling for covariates, the high exposure group had annual changes in $4 \mathrm{kHz}$ emissions of about $0.5 \mathrm{~dB}$ per year. Pure tone audiometric thresholds displayed only slight trends towards increased threshold levels with increasing exposure groups. Some unexpected results were observed, including an apparent increase in DPOAEs among controls over time, and improvement in behavioural thresholds among controls at $6 \mathrm{kHz}$ only.

Conclusions: Results indicate that construction apprentices in their first three years of work, with average noise exposures under $90 \mathrm{dBA}$, have measurable losses of hearing function. Despite numerous challenges in using DPOAEs for hearing surveillance in an industrial setting, they appear somewhat more sensitive to these early changes than is evident with standard pure tone audiometry.
\end{abstract}

$\mathrm{N}$ oise induced hearing loss (NIHL) remains a significant problem in industry, despite substantial research on the mechanisms of damage, availability of standards for acceptable noise exposure levels, and demonstration of effective programmes for hearing loss prevention. ${ }^{1}$ The problem is particularly important in the construction industry where noise levels frequently exceed standards, ${ }^{2}{ }^{3}$ rates of significant hearing loss and workers' compensation claims are epidemic, ${ }^{4}$ and few hearing loss prevention programmes are effectively applied..$^{5}$

The development of noise induced hearing loss has been described primarily on the basis of cross-sectional studies. For instance, in a classic study of current and retired weavers, hearing thresholds were assessed after exposure to highly steady noise exposure for up to 40 years. ${ }^{6}$ A steep linear increase in $4 \mathrm{kHz}$ thresholds was observed for up to about 12 years of exposure, followed by a slowed but continuing increase in mean thresholds. The increases were less rapid at 3 and $6 \mathrm{kHz}$, and at 6,2 , and $1 \mathrm{kHz}$ the increase in mean thresholds were approximately linear up to the 40 years of exposure covered by the study. This pattern of strongly increasing thresholds over the first few years of exposure, followed by a slowing of loss and spreading from 4-6 kHz to lower frequencies, has been observed in numerous crosssectional studies, ${ }^{7-11}$ and is represented in models developed to predict noise induced hearing loss. ${ }^{12-14}$

In a summary of these studies and models, Johnson notes that "...if these studies are halfway correct, the majority of the NIPTS that will occur, will occur in the first 5 years" ${ }^{15}$ In considering the evidence, Johnson concludes that studying the development of early losses using longitudinal studies, especially in previously unexposed populations, is a priority for research. However, the ability to observe small changes over time requires that the study methods be sufficiently sensitive and precise to detect relatively small changes. While the ANSI model predicts changes of $2.0 \mathrm{~dB}$ per year for the median of a population over three years of exposure to $90 \mathrm{dBA},{ }^{14}$ standard pure tone audiometry has a test-retest variability of about $5 \mathrm{~dB},{ }^{16}$ making observation of such small changes challenging.

In recent years, otoacoustic emissions have been explored as a sensitive and objective measure of the function of outer hair cells, ${ }^{17}$ a primary site of noise induced damage to hearing. ${ }^{18}$ Distortion product otoacoustic emissions (DPOAEs) have shown high sensitivity to noise ${ }^{1920}$ and ototoxic drugs. ${ }^{21} 22$ In animal models, early damage identified by OAEs may be predictive of subsequent hearing threshold increases. ${ }^{23}$ In humans, reductions in DPOAEs have been observed in noise exposed ears even with normal pure tone thresholds. ${ }^{24}$ Furthermore, DPOAE test-retest variability is somewhat lower than for audiometry, on the order of $3 \mathrm{~dB} .{ }^{16}$ As a result, DPOAEs have been proposed for hearing surveillance in industry. ${ }^{25} 26$ However, no study has yet prospectively addressed the relation of noise exposure to DPOAE decline and subsequent hearing threshold increase. Measurement of DPOAEs involve measuring the intensity of sound emanating from the inner ear using a microphone probe placed in the ear canal. The emissions are generated in

Abbreviations: DPOAE, distortion product otoacoustic emission; $\mathrm{HPD}$, hearing protection device; HTL, hearing threshold level; NIHL, noise induced hearing loss; $\mathrm{OAE}$, otoacoustic emission 


\section{Main messages}

- Even at average exposure levels under $90 \mathrm{dBA}$, construction apprentices have small but measurable effects of noise exposure on hearing within the first three years of work in the industry.

- Early effects of noise are more readily identified by distortion product otoacoustic emissions (DPOAEs) around $4 \mathrm{kHz}$ with input primaries around $65 /$ $55 \mathrm{~dB}$, than with traditional pure tone behavioural audiometry.

- Quantitative estimation of noise exposure, including the attenuation of exposure due to hearing protection devices, is important in identifying these subtle changes.

response to two "primary" input tones delivered by the same probe. By altering both the level (intensity) and frequency of the primaries, a spectrum of the responsiveness of the cochlea can be assayed across these two dimensions.

To address the issue of the longitudinal decline in hearing during the first few years of noise exposure, we measured pure tone behavioural thresholds and DPOAEs annually among a group of construction industry apprentices over the first three years of their work in the industry, and compared them to a group of "non-exposed" controls. Occupational and non-occupational noise exposures and other NIHL risk factors were also assessed among the cohort. A description of the cohort at baseline, with evidence of the impact of noise exposure, especially previous work related exposure, on both audiometric thresholds and DPOAEs, has been presented elsewhere. ${ }^{27}$

This analysis was designed to evaluate the relations between noise exposure related changes over time for a range of sound frequencies and DPOAE primary intensities, while controlling for covariates that could alter those relations. Our primary a priori hypothesis was that noise dependent changes over time would be evident at $4 \mathrm{kHz}$, a frequency typically associated with early noise induced hearing loss. We further expected that such changes in DPOAEs would be observed first and most significantly at lower levels of primary tone stimulation.

\section{METHODS}

Subjects were recruited from eight construction trades (carpenters, cement masons, electricians, ironworkers, insulation workers, masonry workers, operating engineers, and sheet metal workers) as early as possible after the beginning of their training programme and at least during the first year of training. Control subjects were recruited from among first year doctoral and medical school students at the University of Washington, to help ensure a comparable age distribution, and potential for four years of the study. After they were provided with information about the study goals and procedures, potential subjects were asked to sign informed consent letters approved by the University of Washington Institutional Review Board. Subjects were paid a nominal incentive for each component of the study in which they participated. Details of recruitment and results of the baseline testing have been presented elsewhere. ${ }^{27}$

Subjects were tested at their apprenticeship programme training site or the University of Washington at recruitment, and again at nominally annual follow up times for three subsequent years. At baseline and each follow up, each subject completed a questionnaire probing history for a number of possible risk factors for hearing loss and providing

\section{Policy implications}

- Construction workers, especially those just entering the industry, continue to have a pronounced need for effective hearing conservation programmes, which will help reduce exposure levels, increase the effectiveness of HPD use, and prevent long term loss of hearing.

- Early detection of noise related hearing damage may be more effectively identified using distortion product otoacoustic emissions; however further work is needed to maximise their accuracy, and determine their utility for screening individual workers.

a task based reconstruction of their work activities and exposures, and any non-occupational noise exposures received since the last examination. Audiometric testing was conducted in a mobile, acoustically treated van by audiology technicians who were certified by the Council for Accreditation in Occupational Hearing Conservation (CAOHC), using an automated test sequence at 500, 1000, 2000, 3000, 4000, 6000, and $8000 \mathrm{~Hz}$, after pre-screening for middle ear problems with otoscopy and screening tympanometry. Ears failing the middle ear screen (visual evidence of obstruction or infection, or pressures lower than -100 daPa), or DPOAE data quality (L1/L2 ratio between 8 and $12 \mathrm{~dB}$ ) were excluded from further testing or analysis.

DPOAEs were measured using a commercially available system (Bio-Logic Scout AuDX) in a quiet room (levels generally less than $70 \mathrm{dBA}$ ), verified by periodic sound level monitoring. DPOAEs corresponding to the frequency $2 \mathrm{fl}-\mathrm{f} 2$ were recorded as "DP-Grams" (21 log spaced $\mathrm{f} 2$ frequencies between 1031 and $10028 \mathrm{~Hz} ; \mathrm{f} 2 / \mathrm{fl}=1.2 ; \mathrm{Ll}=65 \mathrm{~dB}$ SPL; $\mathrm{L} 2=\mathrm{L} 1-10)$. At seven $\mathrm{f} 2$ frequencies chosen to approximate the audiometric test frequencies, DPOAEs also were recorded as functions of increasing stimulus level ( $\mathrm{f} 2 / \mathrm{f} 1=1.2$; $\mathrm{Ll}=35-80 \mathrm{~dB}$ SPL in $5 \mathrm{~dB}$ steps; $\mathrm{L} 2=\mathrm{Ll}-10)$. For the longitudinal analysis, DPOAE I/O functions at 2, 3, 4, and 6 $\mathrm{kHz}$ were emphasised. Noise floors, sampled at $\pm 50 \mathrm{~Hz}$ of the 2fl-f2 frequency, were acceptably low and response levels were analysed without adjustment or censoring in relation to the noise floor.

Exposure groups were defined on the basis of each subject's trade, using extensive trade specific exposure monitoring results reported elsewhere. ${ }^{28}$ Briefly, 557 full shift dosimeter measurements, collected using one-minute data logging intervals, were collected with simultaneous worker reporting of activities and use of hearing protection devices (HPDs). Full shift equivalent average exposures, $\mathrm{L}_{\mathrm{eq}}$, were calculated and averaged by the individual's trade. HPD adjusted $\mathrm{L}_{\mathrm{eq}} \mathrm{s}$ were also calculated by subtracting $20 \mathrm{~dB}$ from the measured sound level for each minute during which HPD use was reported. The average level of attenuation achieved by a sample of construction workers $(n=44)$ on whom we made direct measurements of individual HPD attenuation during typical use in the field was $20 \mathrm{~dB} .{ }^{28}$ The average unadjusted and HPD adjusted $\mathrm{L}_{\mathrm{eq}}$ levels were calculated by trade, and the trades were ranked according to these means. In addition to the control group, two exposure groups (low and high) were then defined by the trade's ranks $1-4$ and 58, respectively. Initial analyses were done using the HPD adjusted $\mathrm{L}_{\mathrm{eq}}$ rankings, as these accounted for use time and approximate HPD attenuation levels, and were assumed to best represent the exposures actually experienced by study subjects.

Longitudinal analyses of changes in hearing level and DPOAEs (frequency specific audiometric threshold or 
frequency and level specific DPOAE intensity) were conducted using mixed effects modelling, with variation between subjects and ears within subject treated as random effects. Predictors of primary interest were time since baseline exam and noise exposure group, the definitions of which are given above. Adjustments also were made for covariates thought to be correlated with both hearing outcomes and occupational noise exposure. These adjustment variables, described in table 3, are gender, baseline age, occupational noise exposure prior to baseline, and mean of baseline audiometric thresholds at 3, 4, and $6 \mathrm{kHz}$. Various measures of non-occupational noise exposure were also considered, but because they were highly correlated with occupational noise exposure, and did not further contribute to the model, were omitted from the final analyses. Tests for interaction between each of these variables and time since baseline were then performed at all $4 \mathrm{kHz}$ hearing outcomes. Only the trade based exposure groups showed significant evidence of interaction. In other words, the model predicts a different yearly change in hearing outcomes for each trade exposure group, but not for each level of the other covariates. Baseline hearing threshold categories were entered as a continuous variable (coded 0,1 , and 2), because inclusion of categorical dummy variables did not improve the model fit, or substantially alter the results. The model, represented in the equation below, was fit to all audiometric and OAE outcomes analysed in this study.

$$
\begin{aligned}
y_{i j t}=\beta_{0} & +\sum_{2} \beta_{a d j} x_{a d j}+\beta_{t} x_{t} \\
& +\sum_{k=1}^{2}\left(\beta_{g, k} x_{g, k}+\gamma_{k} x_{t} x_{g, k}\right)+b_{i}+e_{i j}+\varepsilon_{i j t}
\end{aligned}
$$

where:

$i$ indexes subject

$j$ indexes ear within subject

$t$ indexes time from baseline

$y_{i j t}=$ hearing outcome for subject $i$ on ear $j$ at time $t$

$x_{t}=$ years since baseline

$x_{g, k}=$ indicator variable for exposure group $k$

$x_{a d j}=$ vector of adjustment variables

$b_{i}=$ subject random effect, a $\mathrm{N}\left(0, \sigma_{i}^{2}\right)$ variable, capturing between-subject variability

$e_{i j}=$ ear random effect, a $\mathrm{N}\left(0, \sigma_{i j}^{2}\right)$ variable, capturing within-subject, between-ear variability

$\epsilon_{i j t}=$ residual, a $\mathrm{N}\left(0, \sigma_{e}^{2}\right)$ variable, capturing within-subject, within-ear variability

$\beta$ s are main effect coefficients

$\gamma$ s are interaction term coefficients

Results of these models are displayed as the estimated effect for the time-exposure group interaction, $\gamma_{g, k}$, over the range of frequencies or intensities for each outcome. Models for the a priori hypothesised effects (DPOAE at $\mathrm{f} 2=4 \mathrm{kHz}$ and $\mathrm{Ll}=40$ and $65 \mathrm{~dB}$ ) are given in full.

\section{RESULTS}

Table 1 shows selection of the cohort for this analysis. Of the 434 subjects (840 ears) included in the baseline analysis, 336 (652 ears) had at least one follow up examination, and 221 (432 ears) had all three follow up tests. Results included in the current analysis are those subjects (or ears) with at least one follow up, after excluding individuals $(n=8)$ or ears $(\mathrm{n}=20)$ with middle ear problems or DPOAE data quality problems. The final dataset thus included 328 subjects (632 ears).

Table 2 gives full shift time weighted average unadjusted and HPD adjusted noise levels by trade. Trade specific mean exposures, unadjusted for HPD use, ranged from $90.7 \mathrm{dBA}$ (ironworkers) to $81.8 \mathrm{dBA}$ (insulation workers). After accounting for HPD use, the average reduction in noise level was only about $3 \mathrm{~dB}$, because of the significant amount of time in which the devices were not worn. However, this reduction was highly variable by trade, with an average attenuation of almost $11 \mathrm{~dB}$ for operating engineers and only $0.7 \mathrm{~dB}$ for insulation workers. As a result, the ranking of trades by unadjusted compared to HPD adjusted levels was substantially different. Using HPD adjusted levels, the high exposure group consisted of ironworkers, carpenters, electricians, and cement masons, while the low exposure group included masons, insulation workers, sheet metal workers, and operating engineers. When unadjusted levels were used, cement masons and electricians dropped to the low exposure group, and operating engineers and masonry workers moved up to the high exposure group.

Table 3 gives demographics, including hearing level at baseline, by exposure group. Follow up was very good, with an average of $3.4(0.8)$ exams per subject, which was consistent across exposure groups. Apprentices were predominantly male, while roughly half of all graduate students were female. The age of the cohort was 27.5 (6.4) years at baseline, and was nearly identical in all three groups, although the range of ages among apprentices was larger than in students. Three quarters of the apprentices reported a year or more of work in noisy occupations, including construction work, prior to baseline; this statistic was only somewhat lower among students (60\%). Non-occupational noise exposure-primarily shooting firearms or riding motorcycles-was also very common, though considerably more common among apprentices (81\%) than students $(43 \%)$. As described in detail in an earlier report, ${ }^{27}$ hearing level was worse among apprentices at baseline, presumably due to a combination of factors including previous occupational and non-occupational noise exposures.

Table 4 presents audiometric data and DPOAE results at selected levels $(\mathrm{Ll}=40$ and $65 \mathrm{~dB})$ and frequencies $(2,3,4,6$ $\mathrm{kHz}$ ) by group and year of follow up. Note that poor or declining hearing is represented by high or increasing audiometric thresholds, and small or decreasing DPOAE amplitudes. At baseline, poorest hearing thresholds were observed in all groups at $6 \mathrm{kHz}$. DPOAE levels tended to decrease with increasing frequency. Controls tended to have better hearing thresholds and slightly larger emissions than the low and high exposure groups, even at baseline.

Only very slight trends over time in hearing threshold levels (HTLs) and DPOAEs were evident in this crude analysis. The variation in response is very large with standard deviations in the order of 10-15 dB for audiometric HTL and 7-10 dB for DPOAEs. Very little trend was observed among apprentices for the lower level $(\mathrm{Ll}=40 \mathrm{~dB})$ DPOAE, while apprentices display a slight downward trend in the higher level DPOAEs at 3,4, and $6 \mathrm{kHz}$. These results are displayed for $4 \mathrm{kHz}$ thresholds and DPOAEs in fig 1. A clear separation in results between the students and controls was observed, although very little meaningful trend over time was seen for audiometric thresholds or DPOAEs at $\mathrm{Ll}=40 \mathrm{~dB}$. At $\mathrm{Ll}=65 \mathrm{~dB}$, slight downward trends in DPOAEs were seen for the exposed groups, and a slight upward trend appeared to be present in the controls. The large variances in relation to the slight trends underscore the difficulty of observing changes within the period of time studied.

Longitudinal models were run with random effects for subject and ear and fixed effect covariates for gender (male/ female), age at baseline $(\leqslant 30,>30)$, hearing level at baseline (mean threshold at 3, 4, and $6 \mathrm{kHz}, \leqslant 10,>10-20,>20 \mathrm{~dB}$ ), and occupational exposure prior to baseline (yes/no). Exposure was considered as time (years) since baseline, exposure group (control, low, high) and the interaction of 
Table 1 Subject selection for longitudinal analysis

\begin{tabular}{|c|c|c|c|c|c|c|}
\hline & \multicolumn{2}{|c|}{ Apprentices } & \multicolumn{2}{|l|}{ Controls } & \multicolumn{2}{|l|}{ Total } \\
\hline & Subjects & Ears & Subjects & Ears & Subjects & Ears \\
\hline Included in baseline analysis & 372 & 720 & 62 & 120 & 434 & 840 \\
\hline With 1 follow up & 276 & 536 & 60 & 116 & 336 & 652 \\
\hline With 2 follow ups & 228 & 445 & 56 & 108 & 284 & 553 \\
\hline With 3 follow ups & 169 & 331 & 52 & 101 & 221 & 432 \\
\hline Excluded* & 8 & 18 & 0 & 2 & 8 & 20 \\
\hline Included in current analysis & 268 & 518 & 60 & 114 & 328 & 632 \\
\hline
\end{tabular}

time since baseline and exposure group. Although the trends were similar for exposure using HPD adjusted, or unadjusted rank groupings, the results for HPD adjusted ranks were considerably clearer, and are presented here. The coefficients and $95 \%$ confidence intervals for exposure (interaction of time and group) are shown for audiometric thresholds (fig 2A), DPOAE magnitude across 21 frequencies (at $\mathrm{Ll}=65 \mathrm{~dB}$ ) (fig 2B), and across primary intensities $(\mathrm{Ll}=35$ to $80 \mathrm{~dB})$ for frequencies of $2,3,4$, and $6 \mathrm{kHz}$ (fig $2 \mathrm{C}-\mathrm{F}$ ).

Pure tone thresholds at 2, 3, and $4 \mathrm{kHz}$ changed little with time, although the ordering of the coefficients for the three groups suggests a slight worsening of hearing (increasing thresholds) with higher exposures (fig 2A). The trends for the high exposure and control groups are statistically significantly different in this frequency range, although at no frequency is that for high exposed subjects significantly greater than 0 . At $6 \mathrm{kHz}$, the control group shows a significantly increased threshold over time.

The pattern of time related change in DPOAEs across frequencies (for $\mathrm{Ll}=65 \mathrm{~dB}$ ) shows little difference between groups at the lower frequencies, a significant separation between controls and the two exposed groups around 3$4 \mathrm{kHz}$, and non-significant and more variable separation at higher frequencies (fig 2B). At very high frequencies (8$10 \mathrm{kHz}$ ), there is once again a significant separation, with suggestions of increasing DPOAEs over time (improving response) among controls, and of decreasing DPOAEs (weaker response) in the exposure groups.

Changes in the growth functions (response at a given frequency over increasing intensity levels of the input primaries) are most clearly shown for the $4 \mathrm{kHz}$ primaries (fig 2E). The effect of time in the high exposure group is significantly more negative (faster loss of function) than in the control group for $\mathrm{Ll}$ in the range of 50 to $75 \mathrm{~dB}$, and significantly lower than 0 from 45 to $70 \mathrm{~dB}$. The change in DPOAEs over time for the low exposure group is intermediate between the controls and high exposure group, indicating a relatively consistent exposure-response gradient. The positive coefficient (increase in DPOAE magnitude over time-that is, better hearing) for the control group is an unexpected result, though it is important to note that it is significantly greater than 0 only at $70 \mathrm{~dB}$.

At 3 and $6 \mathrm{kHz}$, there is very limited evidence of an exposure gradient for change in DPOAEs over time. In the mid levels (45-75 dB), there remains a significant difference between the $3 \mathrm{kHz}$ DP changes among controls and apprentices, although there is not much difference between the high and low exposure groups (fig 2D). The picture is even less pronounced at $6 \mathrm{kHz}$, with differences between controls and apprentices only observed at the high stimulus levels $(\geqslant 65 \mathrm{~dB}$ ) (fig $2 \mathrm{~F}$ ). On the other hand, although at $3 \mathrm{kHz}$ the effect of time was significantly lower than 0 only in the high exposure group for $\mathrm{Ll}$ in the range from 55 to $65 \mathrm{~dB}$, at $6 \mathrm{kHz}$ it was consistently below 0 in both exposure groups for almost all Ll levels. At $2 \mathrm{kHz}$, no differences between groups were observed, and the effect of time was significantly lower than 0 only in the low exposure group for $\mathrm{Ll}=55$ and $65 \mathrm{~dB}$ (fig 2C).

Table 5 gives the full models for $4 \mathrm{kHz}$ audiometric thresholds and DPOAE with $\mathrm{Ll}=40$ and $65 \mathrm{~dB}$. As noted in the methods section, these particular models were selected based on a priori hypotheses, and not because they necessarily showed the strongest results. The audiometry model indicates higher thresholds (worse hearing) for low and high exposure groups in comparison to controls. By combining the modelled effect of years of follow up with the interaction of follow up time and exposure group, it is evident that the controls have slightly (although not significantly) decreasing thresholds (improving hearing) over time, while there is very little discernable change with time for the two exposure groups. At a low level stimulus $(\mathrm{Ll}=40 \mathrm{~dB})$ there is no discernable change over time at $4 \mathrm{kHz}$, while at $65 \mathrm{~dB}$, there is a significant change over time of about -0.4 and $-0.5 \mathrm{~dB}$ per year (sum of coefficients for years since baseline and interaction terms) for the low and high exposure groups, respectively. These changes are significantly different from 0 , and from the modelled change in the control group. The

\begin{tabular}{|c|c|c|c|c|c|}
\hline \multirow[b]{2}{*}{ Trade } & \multirow{2}{*}{$\begin{array}{l}\text { Number } \\
\text { shifts }\end{array}$} & \multicolumn{2}{|c|}{ HPD adjusted } & \multicolumn{2}{|c|}{ Unprotected } \\
\hline & & Mean (SD) & Rank & Mean (SD) & Rank \\
\hline Ironworkers & 37 & $89.5(6.3)$ & 1 & $90.7(5.5)$ & 1 \\
\hline Carpenters & 81 & $86.2(6.9)$ & 2 & $89.3(4.5)$ & 2 \\
\hline Electricians & 230 & $86.2(6.2)$ & 3 & $86.7(5.5)$ & 6 \\
\hline Cement masons & 31 & $85.0(7.8)$ & 4 & $87.7(5.6)$ & 5 \\
\hline Masonry workers & 73 & $84.4(7.0)$ & 5 & $88.5(6.7)$ & 3 \\
\hline Insulation workers & 23 & $81.1(4.2)$ & 6 & $81.8(3.8)$ & 8 \\
\hline Sheet metal & 43 & $78.8(8.0)$ & 7 & $85.7(4.2)$ & 7 \\
\hline Operating engineers & 33 & $77.3(9.0)$ & 8 & $88.1(6.0)$ & 4 \\
\hline
\end{tabular}


Table 3 Demographic characteristics of study subjects

\begin{tabular}{lllll}
\hline & Controls & Low exposure & High exposure & Total \\
\hline Number of subjects analysed & 60 & 122 & 146 & 328 \\
Number of exams, mean (SD) & $3.7(0.6)$ & $3.3(0.8)$ & $3.4(0.8)$ & $3.4(0.8)$ \\
Male, $\mathrm{n}(\%)$ & $32(53.3)$ & $112(91.8)$ & $240(88.9)$ & $272(82.9)$ \\
Age, mean (SD) & $27.3(4.2)$ & $27.2(6.7)$ & $27.8(6.8)$ & $27.5(6.4)$ \\
Prior occupational noise, $\mathrm{n}(\%)$ & $36(60)$ & $102(79.6)$ & $112(76.7)$ & $250(76.1)$ \\
Prior non-occup. noise, $\mathrm{n}(\%)$ & $26(43.3)$ & $101(81.4)$ & $118(80.8)$ & $245(74.7)$ \\
Number of ears analysed & 114 & 238 & 280 & 632 \\
BL audiogram $\leqslant 10 \mathrm{~dB}, \mathrm{n}(\%)^{*}$ & $78(68.4)$ & $132(55.5)$ & $153(54.6)$ & $363(57.4)$ \\
BL audiogram 10-20 dB, $\mathrm{n}(\%)$ & $31(27.2)$ & $73(30.7)$ & $74(26.4)$ & $178(28.2)$ \\
BL audiogram $>20 \mathrm{~dB}, \mathrm{n}(\%)$ & $5(4.4)$ & $33(13.8)$ & $53(18.9)$ & $91(14.4)$ \\
\hline${ }^{*}$ Mean $\mathrm{HL}$ at $3,4,6 \mathrm{kHz}$. & & & &
\end{tabular}

models also indicate that, as expected, higher thresholds and lower DPOAEs were associated with previous occupational noise exposure, older age, and higher thresholds at baseline. Male gender was also associated with worse hearing (higher thresholds and lower DPOAE amplitudes), but not significantly.

\section{DISCUSSION}

Demonstration of changes in hearing function over relatively short periods of occupational noise exposure is challenging because shifts are small and standard audiometric techniques have test-retest variability large enough to mask them. In order to address the development of noise related changes in hearing, a cohort of construction industry apprentices was recruited at the beginning of their careers, and followed annually for three years using standard pure tone audiometry coupled with distortion product otoacoustic emission measurements. To control for any time trends in the measurement techniques employed, a group of graduate students in the beginning of their educational programmes were recruited and similarly followed. The primary results of the longitudinal analysis are presented here, and show that small but significant decrements over time in DPOAEs were evident among the construction trades at 3, 4, and $6 \mathrm{kHz}$, with more highly exposed trades having a larger decrease at $4 \mathrm{kHz}$ than trades with lower exposures. The magnitude of the observed change, about $-0.5 \mathrm{~dB}$ per year, depending on frequency and level of the primary input tones, was smaller than expected but indicative of the potentially long term damaging effect of noise on hearing, even within the first three years of work in the trades. While patterns of change over time in audiometry were generally consistent with noise related increases in thresholds, the changes were extremely small, and not significantly different from 0 . These findings were consistent with our a priori hypotheses-that DPOAEs would be more sensitive to noise exposure than HTLs, and that the largest response would be in the range of $3-6 \mathrm{kHz}$. Furthermore, these findings extend and strengthen the findings from this cohort analysed cross-sectionally at baseline. ${ }^{27}$

Among the most significant challenges for this study is the accurate determination of noise exposures over time for a large cohort of subjects who were working at numerous worksites, and in an industry known for its highly intermittent and variable exposures. ${ }^{58}$ The study benefited greatly by the collection of a very large and comprehensive dataset describing noise exposures among commercial construction workers. In this initial analysis, exposure was based on a rank ordering of the trades, rather than individual level exposure estimates. Although individual task based assessments could be more specific for the activities done by each individual, a large degree of misclassification would also be introduced through this method. ${ }^{29}$ The grouping of exposures into relatively crude categories reduces misclassification, but may also attenuate the underlying exposure-response relations. Additional analyses comparing alternative methods of exposure estimation for this cohort are forthcoming.

Exposure estimation can be particularly inaccurate among groups in which the use of personal protective equipment is variable. ${ }^{28}$ In addition to differences in individual fitting of hearing protection devices (HPDs), the time of use, and the exposure level during times of use and non-use are crucial elements in determining the actual dose received by the

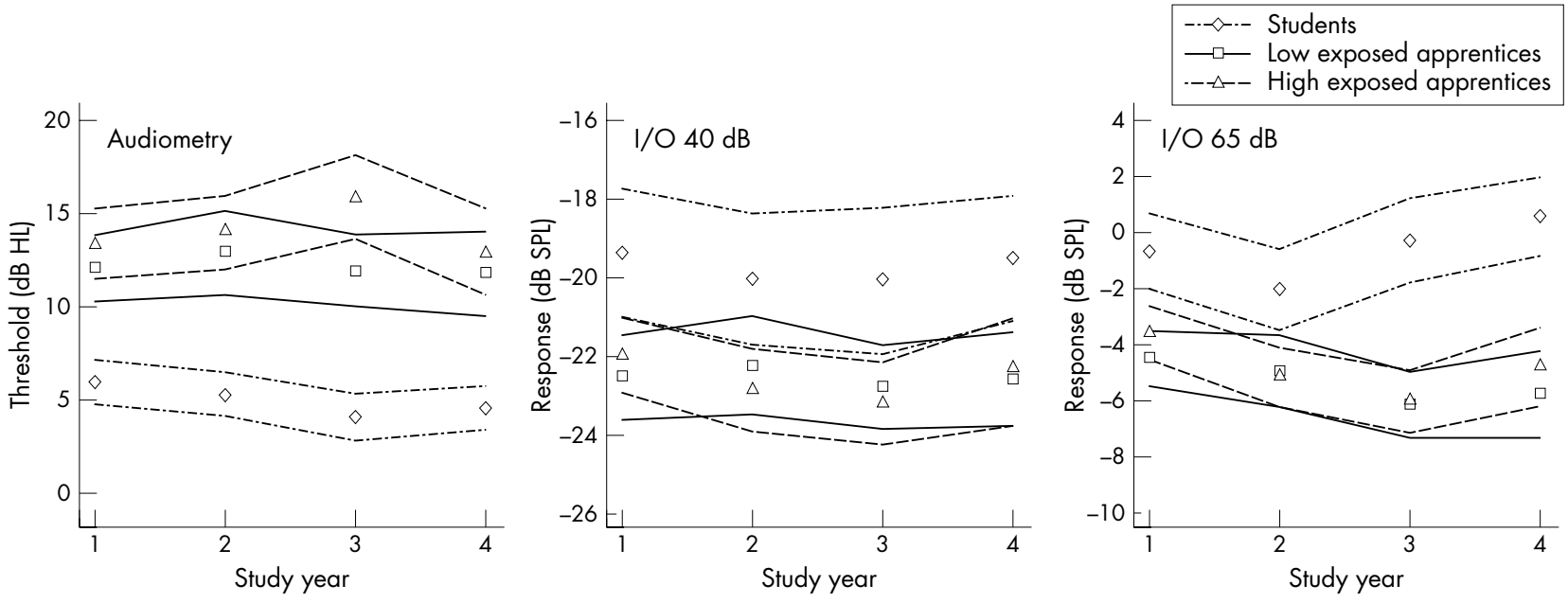

Figure 1 Mean $(95 \% \mathrm{Cl}) 4 \mathrm{k}$ outcomes over time comparing students and low and high exposure groups of construction apprentices. 


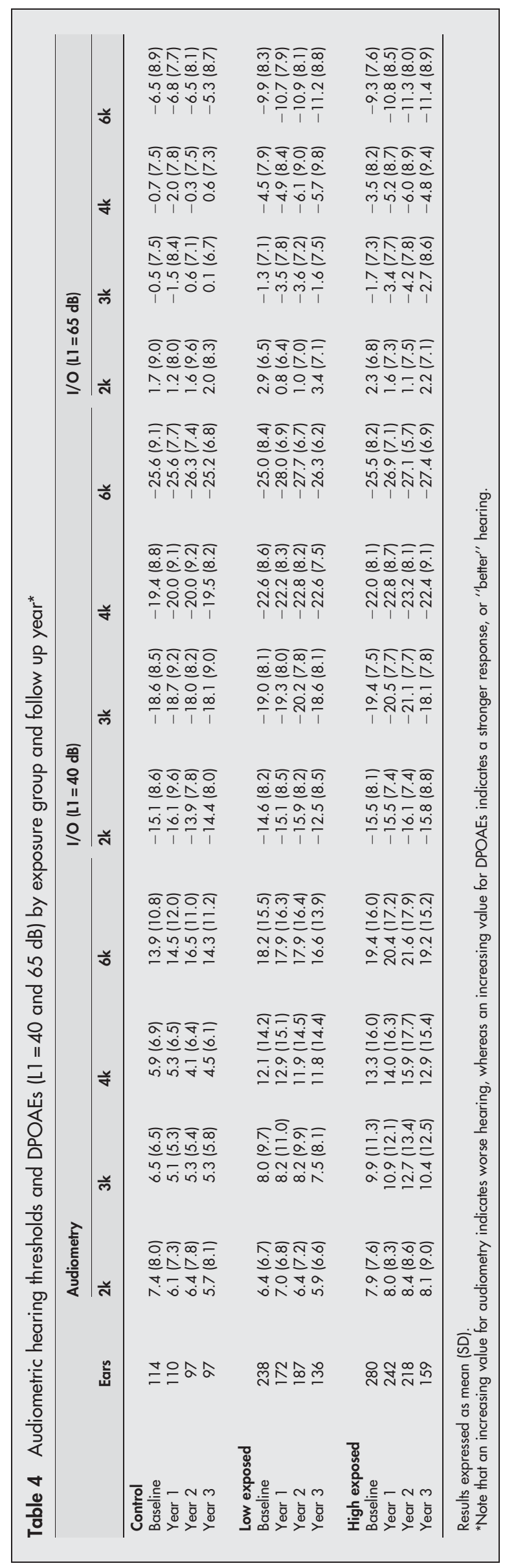

individual. By combining data logging dosimetry with individual HPD use, self-reporting and direct measurements of attenuation from a small number of subjects, we were able to adjust trade specific average exposures to better reflect the true exposure levels. Because HPD use varied greatly by trade, the rank ordering of trades by HPD adjusted, and unadjusted levels was very different. As a result, the HPD adjusted ranks showed a much clearer dose-response relation than was the case when HPD use data were not utilised.

The high exposure group had estimated average exposures between 85 and $90 \mathrm{dBA}, \mathrm{L}_{\mathrm{eq}}$. At these levels of exposure, the decrease in median audiometric thresholds at $4 \mathrm{kHz}$ over the first three years of exposure would be 0.9 and $2.0 \mathrm{~dB}$ per year, respectively, according to a widely accepted model for predicting noise induced hearing loss. ${ }^{14}$ Our results, indicating almost no change in HTLs over a three year period of observation are not consistent with this model. The ANSI model depends on interpolation of hearing thresholds for changes in hearing resulting from exposure periods of less than 10 years, and may not correctly represent the true progress of NIHL over this relatively short period of exposure. Furthermore, the ANSI model predicts a non-linear change in hearing, with lower expected losses after some period of exposure. The exposed subjects in this study reported an average of 2.2 (3.2) years of previous construction experience at baseline, and 3.8 (6.0) years of previous noisy nonconstruction work experience. ${ }^{27}$ Thus, the expected change in hearing from the ANSI model would best be predicted by the interval 3-6 years after first exposure. Over this interval, the ANSI model predicts a change of 0.4 and $0.9 \mathrm{~dB}$ per year at 85 and $90 \mathrm{dBA}$, respectively. These lower predicted changes over the 3-6 year interval are closer to the changes in audiometric thresholds seen in the high exposure group of subjects.

Although average exposure levels were between 85 and 90 $\mathrm{dB}$, even accounting for HPD use, it is possible that the highly intermittent exposures found in the construction industry produces a different course of hearing damage than would continuous exposures. In some studies, intermittent exposures have produced less hearing loss than steady state exposures of the same magnitude, thus providing some justification for the $5 \mathrm{~dB}$ exchange rate exposure metric used by the US OSHA. ${ }^{1}$ However, this model has been largely rejected by most international bodies, and replaced with the equal energy hypothesis. It would appear more likely that the less than expected change in HTLs in our cohort has to do with the relatively short duration of follow up and the variability in the hearing measurements.

Although the results are consistent with our a priori hypotheses, they raise a number of significant issues. One of these is the apparent improvement in HTLs and increase in DPOAEs (for example, improvement in hearing functions) over time among the control subjects. The apparent improvement is of a similar magnitude as the decrease in function among the exposed groups. The most probable explanation for such a change would be alterations in equipment or technique in conducting the tests. Audiometry was conducted by an experienced audiometric testing company using equipment and sound test enclosures meeting OSHA standards. No systematic changes in equipment output or test environment characteristics could be identified during this follow up period. Some data were collected towards the end of the study using portable audiometry administered in a quiet room, which was not compliant with OSHA or ANSI requirements for audiometric testing. However, when these results were excluded from the analysis, no significant change in results was observed. We also stratified our cohort by those tested during the first half of the first year to test for a learning effect among our testing personnel. Again, this could not explain away the apparent improvement. 

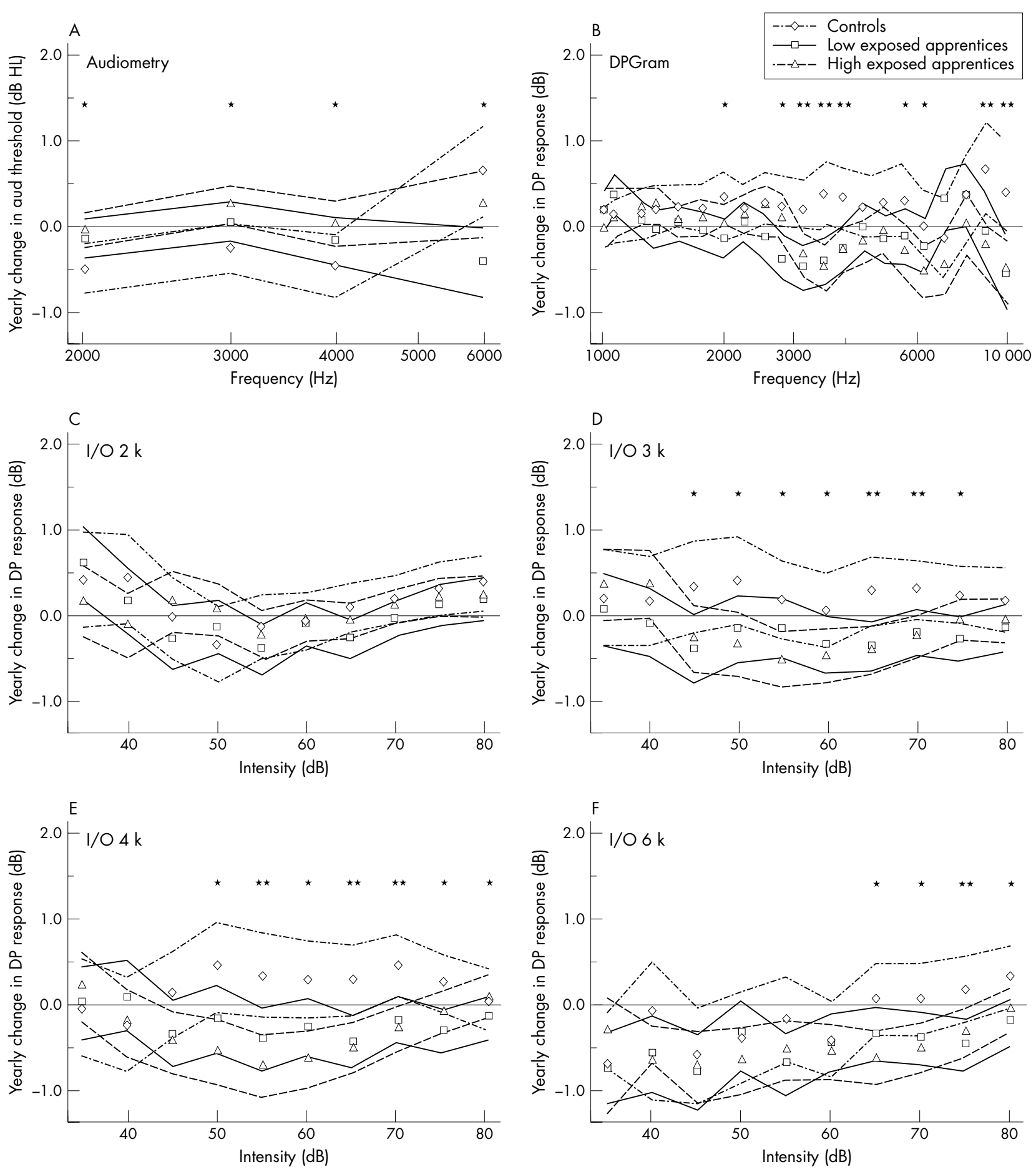

Figure 2 Coefficient $(95 \% \mathrm{Cl})$ for interaction of exposure group and time from mixed effects models for all outcome measures (see text for model specification). Groups shown are controls, low exposed apprentices, and high exposed apprentices. A positive coefficient for audiometric thresholds (A) and a negative coefficient for DPOAEs (B-F) indicate a worsening of hearing function over time. The number of asterisks at a particular outcome represents the number of exposure groups (1 or 2 ) that are significantly different from controls.

Although a learning effect could be present for the behaviourally delivered audiometric thresholds, the DPOAEs have the advantage of being objective and nonbehaviourally dependent measurements. We checked to see if temporary threshold shifts due to reported noise exposure within 16 hours of the examination could explain the results, but no effect on the results was observed. As a result, the apparent improvement in hearing among controls remains unexplained. If the improvement in hearing measurement were similar between the controls and construction apprentices, then the noise related changes among the apprentices would be under-estimated by the observed results.

A larger fraction of the apprentices (28\%) than controls (3\%) dropped out of the study after enrolment. The study cohort and those that dropped out or were excluded had very similar hearing levels at baseline. In the high exposure group, a slightly higher percentage of the studied cohort, compared to the excluded group, had audiometric thresholds greater 
Table 5 Model coefficients $(95 \% \mathrm{Cls})$ at $4 \mathrm{kHz}^{*}$

\begin{tabular}{lrrr}
\hline & Audiometry & I/O $40 \mathrm{~dB}$ & I/O 65 dB \\
\hline Years since baseline & $-0.46(-0.83$ to -0.09$)$ & $-0.23(-0.76$ to 0.30$)$ & $0.29(-0.10$ to 0.68$)$ \\
Low exposure group & $2.83(0.11$ to 5.55$)$ & $-1.05(-2.99$ to 0.89$)$ & $-0.46(-2.32$ to 1.40$)$ \\
High exposure group & $3.15(0.54$ to 5.76$)$ & $-0.66(-2.52$ to 1.20$)$ & $0.29(-1.49$ to 2.07$)$ \\
Follow up years * low exposure group & $0.30(-0.16$ to 0.76$)$ & $0.32(-0.35$ to 0.99$)$ & $-0.72(-1.23$ to -0.21$)$ \\
Follow up years * high exposure group & $0.50(0.04$ to 0.96$)$ & $0.02(-1.86$ to 1.90$)$ & $-0.80(-1.29$ to -0.31$)$ \\
Previous occupational noise & $1.25(-0.83$ to 3.33$)$ & $-1.19(-2.54$ to 0.16$)$ & $-0.88(-2.25$ to 0.49$)$ \\
Age $>30$ & $3.92(1.94$ to 5.90$)$ & $-1.10(-2.39$ to 0.19$)$ & $-2.64(-3.93$ to -1.35$)$ \\
Baseline audiometry $(0=\leqslant 10,1=10-20,2=>20)$ & $12.40(11.32$ to 13.48$)$ & $-3.44(-4.15$ to -2.73$)$ & $-5.22(-5.89$ to -4.55$)$ \\
Male gender & $1.26(-1.23$ to 3.75$)$ & $-2.31(-3.94$ to -0.68$)$ & $-3.47(-5.12$ to -1.82$)$ \\
\hline
\end{tabular}

*Note that a positive coefficient for the audiometry model indicates association with worse hearing, whereas a positive coefficient for the OAE models indicates association with stronger response, or "better" hearing.

than $20 \mathrm{~dB}(19 \% \quad v 10 \%)$. Among the controls and low exposure groups, the included and excluded subjects had almost identical rates of hearing loss at baseline. We conclude that there is little evidence of bias in the results due to differential follow up. It is also reassuring that in the current analysis all four covariates (previous occupational noise, older age, baseline audiometry, and male gender) which represent aspects of greater exposure to noise, entered the audiometry and DPOAE models with effects in the expected direction.

DPOAEs have shown promise as a sensitive early indicator of hearing damage induced by noise exposure, and may therefore have potential surveillance applications as part of a hearing loss prevention programme. ${ }^{25} 260$ The results presented here support this suggestion, showing that, at least on average, significant decreases in DPOAE magnitudes were observed in an exposure dependent way over a period of less than three years. While this finding reinforces the obvious problem that construction workers continue to be exposed to unsafe levels of noise, and without adequate protection, the application of DPOAEs to identify individuals with preclinical evidence of noise related damage requires additional investigation. Although the DPOAEs measured for this study had a higher precision than the pure tone thresholds (test-retest standard deviations of about $3 \mathrm{~dB} v 5 \mathrm{~dB}$ ), this level of precision still may be inadequate for individual level surveillance over short time periods.

A further question is whether the changes observed in DPOAEs can be used to predict future changes in HTLs, on either an individual or group basis. However, with the very small changes in DPOAEs, the almost non-existent changes in HTLs, and the very limited follow up time, this study was unable to answer this question. Further follow up of the cohort will help determine if changes in DPOAEs can be used to predict later changes in hearing levels, on either an individual, or group basis. If so, DPOAEs will form an important tool for monitoring hearing damage risk, and preventing noise induced hearing loss.

\section{ACKNOWLEDGEMENTS}

The authors are grateful to Susan Brower, study coordinator for the project, for her endless effort and skill in tracking subjects and ensuring data quality. We are further indebted to the study participants, and the Western Washington Building Trades Apprenticeship Program coordinators for making the study possible. This project was supported by Grant number 5 ROI OH03912 from the National Institute for Occupational Safety and Health of the US Centers for Disease Control and Prevention.

\section{Authors' affiliations}

N S Seixas, B Goldman, L Sheppard, R Neitzel, University of Washington, Department of Environmental and Occupational Health Sciences, Seattle, WA, USA

S Norton, Children's Hospital and Regional Medical Center, Seattle, WA, USA
S G Kujawa, Department of Otology and Laryngology, Harvard Medical School and Massachusetts Eye and Ear Infirmary, Boston, MA, USA

Competing interests: none

\section{REFERENCES}

1 NIOSH. Criteria for a recommended standard: occupational noise exposure. Cincinnati, OH: US Department of Health and Human Services/Centers for Disease Control, National Institute for Occupational Safety and Health, 1998.

2 Neitzel R, Seixas N, Camp J, et al. An assessment of occupational noise exposures in four construction trades. Am Ind Hyg Assoc J 1999;60:807-17.

3 Seixas N, Ren K, Neitzel R, et al. Noise exposure among construction electricians. Am Ind Hyg Assoc J 2001;62:615-21.

4 Daniell W, Fulton-Kehoe D, Cohen M, et al. Increased reporting of occupational hearing loss: workers' compensation in Washington State, 1984-1998. Am J Ind Med 2002;42:502-10.

5 Suter A. Construction noise: exposure, effects and the potential for remediation; a review and analysis. Am Ind Hyg Assoc J 2002;63:768-89.

6 Taylor W, Pearson J, Mair A, et al. Study of noise and hearing in jute weaving. J Acoust Soc Am 1965;38:113-20.

7 Gallo R, Glorig A. Permanent threshold shift changes produced by noise exposure and aging. Am Ind Hyg Assoc J 1964;25:237-45

8 Burns W, Robinson D. Hearing and noise in industry. London: HMSO, 1970.

9 Evans W, Ming H. Industrial noise-induced hearing loss in Hong Kong-a comparative study. Ann Occup Hyg 1982;25:63-80.

10 Thiery L, Meyer-Bisch C. Hearing loss due to partly impulsive industrial noise exposure at levels between 87 and $90 \mathrm{~dB}(\mathrm{~A})$. J Acoust Soc Am 1988;84:651-9.

11 Kamal A, Mikael R, Faris R. Follow-up of hearing thresholds among forge hammering workers. Am J Ind Med 1989;16:645-58.

12 Kryter K. Physiological, psychological and social effects of noise. NASA Ref Pub 1984;1115:276-89.

13 ISO. Acoustics - determination of occupational noise exposure and estimation of noise-induced hearing impairment. Geneva: International Organisation for Standardisation, 1990.

14 ANSI. Determination of occupational noise exposure and estimation of noiseinduced hearing impairment, S3.44-1996. New York: American National Standards Institute, 1996.

15 Johnson DL. Field studies: industrial exposures. J Acoust Soc Am 1991;90:170-4.

16 Hall A, Lutman M. Methods for early identification of noise-induced hearing loss. Audiology 1999;38:277-80.

17 Kemp D. Otoacoustic emissions in perspective. In: Robinette M, Glattke T, eds. Otoacoustic emissions: clinical applications. New York: Thieme, 1997:1-21.

18 Dallos P. The active cochlea. J Neurosci 1992;12:4575-85.

19 Hamernik RP, Ahroon WA, Lei SF. The cubic distortion product otoacoustic emissions from the normal and noise-damaged chinchilla cochlea. J Acoust Soc Am 1996;100(2 pt 1):1003-12.

20 Skellett R, Crist J, Fallon $M$, et al. Chronic low-level noise exposure alters distortion product otoacoustic emissions. Hear Res 1996;98:68-76.

21 Brown AM, McDowell B, Forge A. Acoustic distortion products can be used to monitor the effects of chronic gentamicin treatment. Hear Res 1989;42:143-56

22 Shi Y, Martin W. ABR and DPOAE detection of cochlear damage by gentamicin. J Basic Clin Physiol Pharmacol 1997;8:141-55.

23 Mensh BD, Lonsbury-Martin BL, Martin GK. Distortion-product emissions in rabbit: II. Prediction of chronic-noise effects by brief pure-tone exposures. Hear Res 1993;70:65-72.

24 Lonsbury-Martin B, McCoy $M$, Whitehead M, et al. Otoacoustic emissions: future directions for research and clinical applications. Hear $J$ 1992;45:47-52.

25 Prasher D, Sulkowski W. The role of otoacoustic emissions in screening and evaluation of noise damage. Int J Occup Med Environ Health 1999;12:183-92.

26 Sliwinska-Kowalska M, Zamyslowska-Szmytke E, Szymczak W, et al. Ototoxic effects of occupational exposure to styrene and co-exposure to styrene and noise. J Occup Environ Med 2003;45:15-24. 
27 Seixas N, Kujawa S, Norton S, et al. Predictors of hearing threshold levels and distortion product otoacoustic emissions among noise exposed young adults. Occup Environ Med 2004;61:899-907.

28 Neitzel R, Seixas N. Effectiveness of hearing protection use among construction workers. J Occup Environ Hyg In press.
29 Seixas NS, Sheppard L, Neitzel R. Comparison of task-based estimates with full-shift measurements of noise exposure. AlHA J (Fairfax, Va) 2003;64:823-9.

30 LePage E. Occupational noise-induced hearing loss: origin, characterisation and prevention. Acoustics Australia 1998;26:2-57.

\section{$\mathrm{ECHO}$}

\section{Doctors discover clues in early RA to lost productivity later}

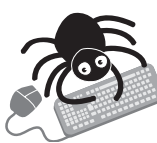

Please visit the Occupational and

Environmental Medicine website [www. occenvmed. com] for a link to the full text of this article. atients with rheumatoid arthritis (RA) should suffer less hardship through work disability now that a Finnish study has identified risk factors that predict loss of productivity and those patients needing early aggressive treatment most.

Global assessments of the severity of RA $\geqslant 50$ by patients and doctors and HAQ scores $\geqslant 1$ at baseline were the disease variables, plus low education and older age, that predicted lost productivity in multivariate analysis in the controlled prospective follow up FIN-RACo trial.

Treatment with combination disease modifying antirheumatic drugs (DMARDs) and prednisolone over two years protected to some extent against lost productivity over treatment with a single DMARD (odds ratio 0.59) at five years' follow up.

Global assessments have seldom been measured before in studies of outcome in RA. HAQ scores showed similar results to previous studies, but here proved a better indicator of lost productivity than single clinical variables. To measure lost productivity more accurately cumulative disability days per patient observation year, proportionate to loss of productivity, were calculated.

Patients with active RA for less than two years, who had not received DMARDs, were assessed according to number of sick days and RA related permanent disability pension. Initially they had sulfasalazine, methotrexate, hydroxychloroquine in combination or sulfasalazine alone; after two years treatment was unrestricted, and 48 patients switched to combination drugs. At entry 80 patients receiving combination and 82 single treatments were working or available for work.

A Puolakka K, et al. Annals of the Rheumatic Diseases 2005;64:130-133. 